\title{
QoS Aware Multipath Threshold Routing for Mobile Ad hoc Networks
}

\author{
M. Ali \\ Caledonian College of \\ Engineering, Oman
}

\author{
B. G. Stewart \\ Glasgow Caledonian \\ University, UK
}

\author{
A. Shahrabi \\ Glasgow Caledonian \\ University, UK
}

\author{
A. Vallavaraj \\ Caledonian College of \\ Engineering, Oman
}

\begin{abstract}
Due to their dynamic topology and lack of central administration, Quality of Service (QoS) provisioning in Mobile Ad hoc Networks (MANET) is a challenging task. QoS is crucial for supporting delay sensitive real-time services in MANETs. Due to the un-predictable behaviour and topology changes of such networks, routing protocols need to quickly and accurately capture and manage the delay, congestion and load at various locations of the network. This paper presents a novel threshold based multipath routing approach for enhanced QoS in MANETs. In this approach, when the available bandwidth of a link decreases below a defined threshold or average load or the forwarding delay at a node increases beyond a defined threshold, traffic is distributed over fail-safe multiple routes to reduce the load at a congested node. Through simulation results, we show that the proposed approach achieves improved QoS in terms of end-to-end delay, packet delivery ratio, and throughput for constant bit rate (CBR) traffic when compared with Optimised Link State Routing (OLSR), a popular single path proactive protocol for MANETs.
\end{abstract}

\section{Keywords}

Ad hoc Networks, Multipath Routing, QoS

\section{INTRODUCTION}

A Mobile Ad hoc Network (MANET) is a transient network formed dynamically by a collection of wireless mobile nodes communicating each other without any fixed infrastructure. In a MANET, the users' mobile devices themselves are the network, which cooperatively provide the functionality usually provided by the network infrastructure such as routers, switches, servers etc. Since there are no dedicated switching nodes in such networks as found in fixed networks, a node has the dual functions of a host as well as a router. Since there is no fixed infrastructure, MANETs are rapidly deployable in scenarios such as disaster recovery, business meetings, student collaborations, communication over rugged terrains etc., where establishing infrastructure networks is either impractical or not cost effective. Since wireless channels are bandwidthconstrained, variable capacity, error-prone and insecure, such networks have significantly lower capacity than traditional wired networks. Moreover, because nodes in such networks are generally portable hand held devices with limited battery power, they have limited processing power which in turn limits services and applications that can be supported by each node. The topologies of MANETs are highly dynamic because nodes may join the network, change their position or leave at any time. High node mobility, bandwidth-constrained wireless links, limited battery power and contention for the shared wireless medium makes routing in MANETs a difficult task. Routing protocols should adapt to such dynamism and continue to maintain connectivity between the communicating nodes in the presence of frequent path breaks caused by mobility and/or node failures. Though real-time multimedia applications could be deployed in MANETs, their inability to support required QoS levels is the main concern. Due to these constraints, special cross layer techniques are required to make the routing protocol QoS aware and congestion adaptive to enable such networks to support delay sensitive real-time applications.

\section{1. Ad Hoc Routing Challenges}

Though MANETs are flexible, convenient and highly desirable in the age of ubiquitous computing, there are many challenges and problems that are yet to be addressed. In traditional infrastructure networks, routing protocols run in specialised nodes which are optimised for that purpose with plenty of resources such as energy, memory, processing power etc. On the other hand, routing protocols in MANETs should run on normal resource-constrained nodes which form a topology that is highly dynamic and unpredictable. This highly dynamic nature of a MANET that results in frequent and unpredictable changes of network topology, makes routing in such networks a difficult and complex process. MANETs inherit the traditional problems of wireless communication and wireless networking such as: lack of absolute boundaries of the wireless medium, interference from other signals, less reliability of the wireless medium, time-varying and asymmetric properties of the channel, hidden and exposed node problems etc. In addition to these problems, the nature and characteristics that are specific to MANETs such as: multi-hop routing, resource constrained nodes, autonomous operation, unpredictable environment, dynamically changing topology, network scalability etc., make the process more complex. These challenges and complexities make routing one of the most active research areas within the MANET domain [1].

Routing protocols normally used in ad hoc networks can be broadly classified in to table-driven or proactive and ondemand or reactive categories. In table-driven protocols, each node maintains a table of routes to all other nodes in the network. The drawback is that nodes must periodically exchange messages with routing information to keep routing tables up-to-date. Examples of these kinds of protocols are OLSR [2], DSDV [3], and STAR [4]. In on-demand protocols, however, nodes only compute routes when they are needed. Advantage of on-demand protocols is the reduced routing overheads when compared to table-driven protocols. Examples of these kinds of protocols are AODV [5], DSR [6] and TORA [7]. As a consequence, on-demand protocols are more scalable to dynamic, large networks [7]. The MANET topology is also dynamic because nodes move around and may join or leave the network at any time. Nodes that are in transmission range of each other are called neighbors and can directly communicate with each other. However, data sent to another nonneighbouring node is routed through a sequence of multiple hops, with intermediate nodes acting as routers. 


\section{2. Multipath Routing in MANETs}

Shortest single path routing has been the basis for most of the existing routing protocols proposed for MANETs [8]. However studies have shown that single path routing based on minimum hop count metrics may not always provide best performance, especially in MANETs when there are nodes along the route which are congested or have less resource such as bandwidth or residual energy $[8,9]$. In single path routing, the discovered routes may not be reliable because they could easily be broken due to node mobility or unpredictable link conditions, resulting in transmission failures and delay in the network, making the protocol unreliable. Also, the time delay of single path routing to recover from the broken routes may not be acceptable to many time-sensitive applications [10]. With the increase in the number of nodes, performance of single path on-demand routing protocols degrades sharply. In addition to this, the shortest hop metric overburdens nodes which are part of the shortest path that very often lie in the center of the network [11]. Such overloaded nodes get congested and start dropping packets sometimes leading to network partitions. Thus applying load balancing schemes to routing protocols becomes necessary to push network traffic from the center of the network to less congested links for maximizing the network utilization [11].

Further, in shortest path routing, the energy of intermediate nodes, which are responsible for routing packets between many source destination pairs gets depleted quickly resulting in node failures. Effective load balancing and congestion control mechanisms are essential to ensure QoS for delay sensitive real-time applications. To overcome such limitations of single path routing, multipath routing can be used where methods that find multiple multi-hop routes between the source node and destination node are employed. Studies have shown that multipath routing enhances reliability of routing in dynamic networks like MANETs.

In multipath routing, the discovered paths could be linkdisjoint, node-disjoint or both and could be used either simultaneously or one at a time. In the earlier case, the traffic from source to destination is distributed simultaneously using all the paths. The fraction of traffic distributed in each path may be based on some cost metric such as aggregate bandwidth or average residual energy of the path. In the later case, the multipath routing protocol can select one of the discovered paths as the primary path based on some metric and the remaining paths as alternative routes, to be used when the primary path fails. If the paths are not link-disjoint, many routes may pass through a particular link leading to traffic bottleneck at that link. This problem could be solved by considering traffic loads or congestion levels in the routing metric. Load balancing is thus crucial in ad hoc networks employing multipath routing where routing metrics need to accurately capture and distribute the load at various locations of the network to avoid bottlenecks and congestion [10,12].

The advantages of using multipath routing in MANETs include effective load balancing, higher aggregate bandwidth, route resilience, network scalability and fault-tolerance. Spreading of traffic along multiple routes ensures load balancing, and alleviates congestion and delay in the network. When data from an application is routed through multiple simultaneous paths, the aggregate bandwidth of the paths used may satisfy the bandwidth requirements of the application, which may not be the case when the same data is routed along a single path, especially when wireless links operate with limited bandwidth. The higher aggregate bandwidth of a connection leads to smaller end-to-end delay and higher throughput for the application. Further, due to the availability of simultaneous multiple paths from source to destination, multipath routing can provide route resilience as long as at least one of the paths doesn't fail. In multipath routing, radio interference between nodes in multiple routes must be taken into account as it may limit the achievable throughput. However, studies show that using multipath routing in dense ad hoc networks results in better throughput than using single-path routing $[8,9]$. Thus improved routing solutions which encapsulate and integrate many of these required features are necessary.

As a solution to the issues discussed above, this paper presents a QoS aware multipath threshold based routing protocol (QMTR) to achieve enhanced QoS by reducing congestion in MANETs. This is achieved by employing simultaneous multiple paths from source to destination. In this method, when the available bandwidth at a node decreases below a defined threshold or the forwarding delay or average load at a node increases beyond a defined threshold, traffic is distributed over fail-safe multiple routes to reduce the traffic load on a congested node. Our algorithm for finding multipath routes is based on Scalable Multipath On-demand Routing (SMORT) [13], which computes fail-safe multiple paths, providing all the intermediate nodes on the primary path with multiple routes to the destination. The fail-safe multiple paths will include the nodes with most bandwidth and least delay and load. A cross layer approach is followed by which the available bandwidth, forwarding delay and load from the MAC layer are used for taking appropriate routing decisions.

The paper is organised as follows: Section 2 discusses related work in the area of QoS provisioning in MANETs; Section 3 gives the routing metrics used in the proposed protocol; Section 4 outlines the proposed QMTR algorithm; Section 5 presents simulation environment and analysis of some results, and finally Section 6 concludes the paper.

\section{RELATED WORK}

Several publications have approached QoS provisioning in MANETs. End-to-end QoS is particularly important for realtime streaming applications such as VoIP (Voice over IP) and video conferencing. The acceptable QoS levels for such services are normally parameterised in terms of end-to-end delay, delay variation (jitter) and packet loss rate. Several MAC layer QoS provisioning approaches have been published in the literature [e.g. 14, 15]. However MAC layer support alone may not necessarily provide end-to-end QoS for real-time applications as it is limited to the radio range of a node, making higher layer support essential for guaranteed end-to-end QoS for such applications. The primary network layer approach normally used is through QoS aware routing protocols. Several such protocols are presented in the literature [e.g. 16 - 19]. Ondemand (reactive) routing protocols are found to be more suitable in MANET environments compared to table-driven protocols because of their significant advantage of lower routing overheads.

Marina and Das [20] have proposed a multipath version of the popular AODV protocol called AOMDV. It is designed primarily for highly dynamic ad hoc networks where frequent 
link failures and route breaks occur. With multiple redundant paths, new route discovery is needed only when all paths to the destination fail, unlike single path AODV. The AOMDV algorithm finds multiple loop free link disjoint routes in the MANET and performs better in terms of delay, routing load and route discovery time compared to the single path version. However these multiple paths need not satisfy the QoS requirements of the flow as the intermediate nodes taking part in the multiple paths are not selected based on their QoS capabilities.

Souihli et al. [11] proposed a load-balancing mechanism that pushed the traffic further from the geometric center of the network. They also provided a routing metric that takes into account a node's degree of centrality, for both proactive and reactive routing protocols. For nodes using reactive approaches, they characterised the centrality by the size of the routing tables. For proactive approaches, they defined a node's centrality by the size of its multi point relay (MPR) selector list. These mechanisms improve the load distribution and significantly enhance the network performance in terms of average delay and reliability. However, the protocol being single-path has inherent limitations in that the selected path, though far from network center, may not satisfy the bandwidth and QoS requirements of the flow.

Ivascu et al. [16] presented an approach based on a mobile routing backbone (MRB) for supporting QoS in MANETs. The authors aimed to identify the nodes with capabilities and characteristics that would enable them to take part in the MRB and efficiently participate in the routing process. This approach improves network throughput and packet delivery ratio by directing traffic through lowly congested regions of the network that are rich in resources. However, since only one MRB is identified from source to destination, it has the inherent limitations of single path routing discussed in section 1.1 above. Liao et al. [21] proposed a multipath QoS routing protocol which searches for multiple paths for the QoS route, where the multiple paths refer to a network with a source and a sink satisfying a certain bandwidth requirement. The multiple paths collectively satisfy the required QoS. The protocol is suitable for ad hoc networks with very limited bandwidth where a single path satisfying the QoS requirements is unlikely to exist.

Reddy and Raghavan [13] proposed a scalable multipath ondemand routing protocol (SMORT), which reduced the routing overhead incurred in recovering from route breaks by using secondary paths. SMORT computes fail-safe multiple paths, which provide all the intermediate nodes on the primary path with multiple routes to the destination. The proposed protocol is scalable, and performs better even at higher mobility and higher traffic loads, when compared to the disjoint multipath routing protocol (DMRP) and AODV routing protocol.

Rishiwal et al. [22] proposed a QoS based Power Aware Routing (Q-PAR) protocol for MANETs by modifying the DSR protocol. Q-PAR selects an energy-stable and QoS constrained end-to-end path from source to destination. According to the results presented, the protocol showed increased network life time, improved packet delivery ratio and reduced average packet delay.
All of the above developments have attempted to address the problems of QoS provisioning, load balancing, and congestion control individually. The proposed QMTR protocol in this paper attempts to address all these issues in a single protocol which ensures improved QoS in MANETs. The proposed protocol gives all the advantages of multipath routing and also distributes traffic based on the available bandwidth, delay and load of nodes. QMTR integrates a number of methods into a single protocol to provide improved QoS performance which otherwise was achieved using multiple protocols. Hence QMTR may be preferred for MANETS as an easy implementation solution for many routing related issues.

\section{ROUTING METRICS}

This section introduces the various routing metrics employed in the QMTR protocol.

\subsection{Estimation of Available Bandwidth}

We use the mechanism suggested by [23] for calculation of available bandwidth, where the idle period of the wireless channel is used for the calculation of bandwidth. The idle period is an indication of traffic travelling along the mobile nodes as well as their neighbourhoods. During the idle period the mobile nodes can successfully transmit data packets. The available bandwidth can be calculated using:

$$
B W_{A v}=B W_{M a x} *\left(\frac{I d l e_{t}}{\text { Int }_{t}}\right)
$$

where $B W_{M a x}$ is the maximum bandwidth of the link and $I d l e_{t}$ is the idle period of the wireless channel over a time interval Int $t_{t}$ The network allocation vector (NAV) of the virtual carrier sense mechanism of the IEEE 802.11 can judge whether the wireless channel is idle or busy and can be used to monitor the transition of the channel state. In a unit time interval, the period during which the channel changes its state from busy to idle is defined as Busy. Thus Idle $t_{t}$ can be denoted as:

Idle $_{t}=$ Int $_{t}-$ Busy $_{t}$

Substituting the value of $I d l e_{t}$ into Equation (1), $B W_{A v}$ can be calculated [23].

\section{2 Estimation of Node Delay}

The method proposed in [24] is used for estimation of node delay. The Distributed Coordination Function (DCF) of IEEE 802.11 standard uses Request-To-Send (RTS) and Clear-ToSend (CTS) to overcome the hidden node problem and also as a virtual carrier sensing mechanism. It is assumed that each data transmission should first issue RTS and CTS, followed by an acknowledgement (ACK). The DCF employs two inter-frame spaces (DIFS - DCF Inter-Frame Space and SIFS - Short InterFrame Space) for asynchronous data transmission. In the IEEE 802.11 standard, a mobile node that attempts to transmit packets undergoes transition through various states such as Idle, Packet_Arrival, Back-off and Attempt [24]. The forwarding delay $D_{\text {delay }}^{i}$ at a mobile node $i$ which includes MAC contention and transmission delays is calculated using equation (3) (as given in [9]): 


$$
\begin{aligned}
D_{\text {delay }}^{i} & P^{i}{ }_{\text {idle }}(\text { DIFS }) \times\left(\text { DIFS }+ \text { avg } \_t+D A(i)+\right. \\
& \left(1-P_{\text {idle }}^{i}(\text { DIFS })\right) \times(\text { SIFS }+D B(i))+(L / R)
\end{aligned}
$$

where $P^{i}{ }_{\text {idle }}(t)$ is the probability that node $i$ succeeds in sensing the channel is idle for time interval $t$ and is given by:

$$
P^{i} \text { idle }(t)=e^{-\lambda t}
$$

where $\lambda$ is the average packet arrival rate (including neighbor nodes) at mobile node $i$; $D A(i)$ is the expected delay encountered in the transmission attempt state of the node and is given by:

$$
\begin{aligned}
D A(i)= & P^{i} \text { idle }(\text { slot }) \times(\text { RTS }+2 * \operatorname{SIFS}+\text { CTS })+ \\
& \left(1-P^{i}{ }_{\text {idle }}(\text { slot })\right) \times(R T S+2 * \operatorname{SIFS}+D B(i))
\end{aligned}
$$

$D B(i)$ is the expected delay encountered during back-off and is given by:

$$
\begin{aligned}
D B(i)= & {\left[1 /\left\{P^{i} \text { idle }(D I F S) \times P^{i} \text { idle }(\text { slot })\right] \times\right.} \\
& {\left[P^{i}{ }^{i d l e}(D I F S) \times\left(D I F S+a v g \_b t+R T S+2 * S I F S+\right.\right.} \\
& \left.\left.P^{i}{ }_{\text {idle }}(\text { slot }) \times C T S\right)\right]+\left[\left(1-P^{i}{ }_{\text {idle }}(\text { DIFS })\right) \times X\right]
\end{aligned}
$$

$a v g \_b t$ is the average back-off time of transmission and is expressed through:

$$
\begin{aligned}
a v g_{-} b t & =\sum_{n=0}^{4}\left(P^{i}{ }_{\text {idle }}(\text { slot }) \times\left(1-P^{i}{ }_{\text {idle }}(\text { slot })\right)^{n} \times 2^{n-1} \times W\right) \\
& +\left(1-P^{i} \text { idle }(\text { slot })\right)^{5} \times 2^{4} \times W
\end{aligned}
$$

The terms $L$ and $R$ are packet length and data rate respectively; $W$ is the contention window size, and

$$
X=R T S+3 * S I F S+C T S+L+A C K
$$

Since propagation delay of wireless links is very small, it is assumed to be negligible [24].

\section{3 Estimation of Load}

We use the mechanism based on contention window $(\mathrm{CW})$ and queue length proposed in [25] for load estimation in a node. The random number resulting from the binary exponential back-off algorithm used in the DCF is uniformly distributed in a range, called the contention window, the size of which doubles each time the attempt to transmit is delayed, until a maximum size is reached for the range. Hence $\mathrm{CW}$ gives a good indication of contention of the channel. Thus the traffic load around a mobile node can be estimated by calculating the average contention of the channel around that node [25]. Traffic load over a long period is estimated in order to mitigate the effect of traffic bursts. The calculation of $\mathrm{CW}$ of a node is performed every $t$ seconds. The exponential weighted moving average method is applied to the old contention window $C W_{\text {old }}$ and the current contention window $C W_{\text {current }}$ to calculate $\mathrm{CW}$ through:

$$
C W=\alpha \times C W_{\text {old }}+(1-\alpha) \times C W_{\text {current }}
$$

where $\alpha$ is a smoothing constant. To get a good indication of the current condition of a node, $\alpha$ is set to 0.3 , which grants a higher priority to the current CW [25].

The number of packets in the interface queue of a node can be considered as an indicator of the traffic load of the node. A mobile node with more packets in its interface queue indicates the traffic load on the node. Thus the average queue size can indicate a node's traffic load over a long term period. The calculation of the average queue size is updated every $t$ seconds according to the following formula (see [25]):

$$
Q_{\text {len }}=\beta \times \text { qlen }_{\text {old }}+(1-\beta) \times \text { qlen }_{\text {current }}
$$

where qlen denotes the average queue length and qlen $_{\text {current }}$ denotes the current queue length. $\beta$ is another smoothing constant and is set to 0.3 in the simulations. The parameters $\alpha$ and $\beta$ can be any value selected between 0 and 1 . Based on the $\mathrm{CW}$ and queue length, the local load of node $i$ can be calculated as follows:

$$
\operatorname{Load}_{i}=\gamma \times\left(\frac{C W}{M a x C W}\right)+(1-\gamma) \times\left(\frac{Q_{\text {len }}}{M a x Q_{l e n}}\right)
$$

The selection of $\gamma$ is used to balance the effects of the two factors of $\mathrm{CW}$ and queue length. In this work it is set to 0.5 , which grants the same priority to the two features. It is clear that the smaller the values of $Q_{l e n}$ and $C W$ the lower the load and vice-versa [25].

\section{QoS AWARE MULTIPATH THRESHOLD ROUTING (QMTR)}

The QMTR protocol presented in this work is based on SMORT [13], which is a multipath extension to the well known single path AODV protocol. SMORT achieves scalability by using alternate paths which reduce routing overhead generated by additional route discoveries and route error communications which occur in single path on-demand protocols. SMORT computes fail-safe multiple paths which bypass nodes in the primary path and can be used to re-direct data packets when nodes on the primary path move away or fail. It operates with route discovery, route reply and route maintenance phases. SMORT uses special route request and route reply packets structures for computing fail safe multiple paths from source to destination. Route discovery is initiated by a node by flooding a route-request packet into the network, when it requires a route to some destination node. All the intermediate nodes that have a valid path to the destination send a route-reply packet back to source up on receiving the route request. Otherwise, they rebroadcast the request. Only the first copy of the route-request is re-broadcasted, though nodes accept multiple copies. A requestrcvd table stores all route-request copies received at a node. The destination node initiates a route reply process when it subsequently receives the request, by sending a route-reply packet back to the source. In order to enable computation of multiple fail-safe paths, nodes accept multiple copies of the route-request packet [13]. 
The source node eventually starts sending data packets to the destination when it receives the first route. Since the destination replies to multiple copies of the route request, intermediate nodes may receive multiple route reply packets, but they relay only the first reply. The neighbors through which nodes received the route request packet previously are used to relay the reply. Nodes drop extra replies after copying secondary paths carried in them into their routing tables. If all the routes between source and destination nodes fail during a data transmission session, route maintenance phase is invoked to re-establish the connection. Expired routes are deleted from the routing table stored in the nodes. SMORT avoids routing loops during the route-reply phase by using route-reply packets to carry the full path to the destination. The fail-safe multiple paths avoids the need for fresh route discovery in the event of a route/node failure in the primary path. In [13] the authors have presented proofs that the primary path and fail-safe paths are loop-free.

\subsection{Load Distribution}

A simple traffic allocation strategy is employed where all multiple paths are used simultaneously with equal distribution of traffic load. Figure 1 shows an example of the distribution of traffic from source (S) to destination (D) using four fail safe multiple paths P1 to P4. The source can begin sending data to the destination, once the source node has obtained the multiple paths to the destination. Once a load distribution condition arises at an intermediate node, then the traffic is distributed equally along the available multiple paths.

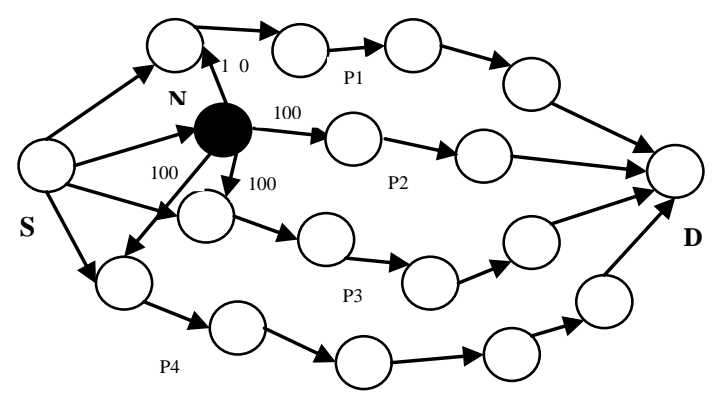

Fig 1: Multipath Load Distribution

For example in Figure 1, let P2 be the primary path through which $\mathrm{S}$ sends data to $\mathrm{D}$. If at time $t_{l}$, a load distribution condition arises at an intermediate node $\mathrm{N}$ (shown as a dark shaded node in Figure 1), then the traffic is distributed (let us say $400 \mathrm{~kb} / \mathrm{s}$ ) equally along all the 4 paths. Thus each path has a load of $100 \mathrm{~kb} / \mathrm{s}$.

\subsection{QMTR Algorithm}

The method of operation of the QMTR algorithm is defined as follows:

1. Estimate the available bandwidth $\left(B W_{A v}\right)$ of the link using $E q(1)$;

2. Estimate the forwarding delay $\left(D_{\text {delay }}^{i}\right)$ at node $N$ using Eq (3);

3. Estimate the load at $N\left(\operatorname{Load}_{i}\right)$ using Eq (11);

4. If $\left\{B W_{A v}<B W_{T h}\right.$ or $D_{\text {delay }}^{i}>D_{T h}$ or $\left.\operatorname{Load}_{i}>\operatorname{Load}_{T h}\right\}$

then, distribute the traffic equally via multiple paths; else, send data through the same path.

The algorithm therefore distributes the traffic from a node $\mathrm{N}$ whenever the available bandwidth at $\mathrm{N}$ is less than the bandwidth threshold $B W_{T h}$ or the forwarding delay at $\mathrm{N}$ exceeds the delay threshold $D_{T h}$ or the average load at $\mathrm{N}$ is more than the load threshold $\operatorname{Load}_{T h}$. The traffic is then distributed equally along the pre-established multiple paths.

\subsection{SIMULATION ENVIRONMENT, RESULTS AND ANALYSIS}

NS-2 [26] has been used to simulate the new protocol. The channel capacity of mobile nodes was set to 2 Mbps. The simulation settings and parameters used in the model are summarised in Table 1.

Table-1: Simulation Parameters

\begin{tabular}{|l|l|}
\hline No. of Nodes & $30,50,70,90$ and 110 \\
\hline Area & 1250 X $1250 \mathrm{~m}$ \\
\hline MAC & IEEE $802.11 \mathrm{DCF}$ \\
\hline Radio Range & $250 \mathrm{~m}$ \\
\hline Simulation Time & $50 \mathrm{~s}$ \\
\hline Traffic Source & CBR \\
\hline Packet Size & $512 \mathrm{~B}$ \\
\hline Mobility Model & Random Way Point \\
\hline Speed & $10 \mathrm{~m} / \mathrm{s}$ \\
\hline Pause Time & $5 \mathrm{sec}$ \\
\hline Rate & $250 \mathrm{~Kb} / \mathrm{s}$ to $450 \mathrm{~Kb} / \mathrm{s}$ \\
\hline
\end{tabular}

In our simulations, $B W_{T h}$ and $\operatorname{Load}_{T h}$ were taken as half of the maximum channel bandwidth and $D_{T h}$ as half of the total MAC delay. Half values were chosen for thresholds intuitively due to the fact that low and high values may trigger late and early load distributions respectively, which are not desirable. The proposed protocol is compared with the OLSR [2] protocol with the same settings and parameters mentioned above. The OLSR protocol is an optimisation of the classical link state algorithm tailored to the requirements of a mobile ad hoc network. The protocol uses multipoint relays (MPRs) that are selected nodes which forward broadcast messages during the flooding process. This technique substantially reduces the message overhead as compared to classical flooding mechanisms, where every node retransmits each message when it receives the first copy of the message. OLSR provides optimal routes which are immediately available when needed.

QMTR was investigated for constant-bit-rate (CBR) traffic using three performance parameters, namely: end-to-end delay, packet delivery ratio (PDR) and throughput. Initially simulations were carried out for network sizes of 30, 50, 70, 90 and 110 nodes, keeping the node speed at $10 \mathrm{~m} / \mathrm{s}$. Then simulations were repeated for varying data rates from $250 \mathrm{Kbps}$ to $450 \mathrm{Kbps}$ in steps of $50 \mathrm{Kbps}$ keeping the number of nodes at 70 and speed at $10 \mathrm{~m} / \mathrm{s}$. The mobility is modelled as the random waypoint model in a square field with a dimension of $1250 \times 1250 \mathrm{~m}$. In all scenarios the number of traffic sessions was kept at 5. Figures 2 to 4 show the results for varying number of nodes while Figures 5 to 8 show the results for varying data rates. 
Figure 2 shows that the end-to-end delay for CBR traffic is considerably less for QMTR compared to OLSR particularly for higher numbers of nodes. This is because the higher the number of nodes, the larger the number of fail-safe multiple paths to the destination. A maximum difference of $100 \mathrm{~ms}$ is seen in end-to-end delay for 90 nodes. In Figures 3 and 4, QMTR shows considerable improvement in PDR and throughput when compared to OLSR. A maximum of $40 \%$ improvement in PDR and 0.4 Mbps improvement in throughput are observed.

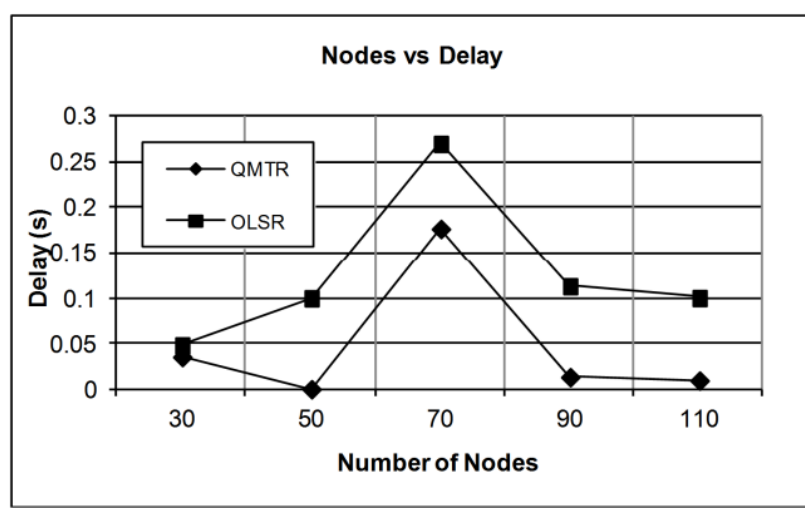

Fig 2: Number of Nodes vs. End-to-End Delay



Fig 3: Number of Nodes vs. Delivery Ratio

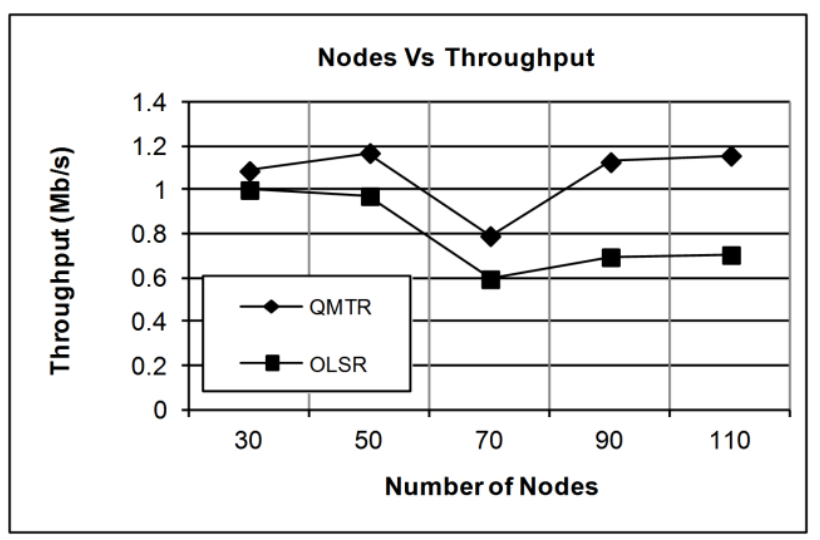

Fig 4: Number of Nodes vs. Throughput
Figure 5 reveals an improvement of $100 \mathrm{~ms}$ in end-to-end delay being observed at $400 \mathrm{Kbps}$ data rate. Figures 6 and 7 respectively show a maximum of approximately $38 \%$ increase in PDR, and a maximum of 0.8 Mbps improvement in throughput.



Fig 5: Data Rate vs End-to-End Delay

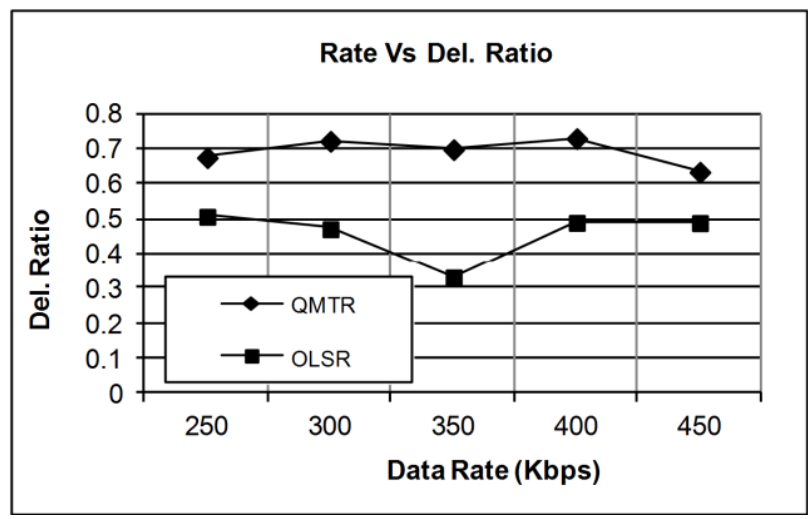

Fig 6: Data Rate vs Delivery Ratio



Fig 7: Data Rate vs Throughput

The reason for the performance improvements may be attributed to the fact that the protocol employs a cross layer approach to capture the available bandwidth, forwarding delay, contention and load at various locations in the network and selects multiple paths which are rich in resources and less congested. This accurately captures and manages congestion throughout the network. It is expected that by avoiding overburdening of nodes which are used for routing of packets, the 
protocol will improve network life time by balancing the load and energy consumption of nodes evenly across the network.

\section{CONCLUSION}

This paper has presented a new QoS aware multipath routing protocol for MANETs. The protocol considers available bandwidth, forwarding delay and load of nodes and distributes traffic through fail-safe multiple paths. Thus the protocol captures bandwidth, delay, load and congestion at various locations in the network and effectively manages them and provides improved QoS to the applications. In the proposed approach when the available bandwidth decreases below a defined threshold or when the forwarding delay or average load at a node increases beyond a defined threshold, traffic is distributed over fail-safe multipath routes to reduce the load at a congested node. The algorithm for finding multipath routes is based on SMORT [13], which computes fail-safe multiple paths, and provides all the intermediate nodes on the primary path with multiple routes to the destination. Simulation results have shown that the protocol achieves better PDR and throughput with reduced end-to-end delay when compared with the OLSR protocol. Since the protocol uses fail-safe multiple paths for all the intermediate nodes on the primary path, the routing overhead may be higher which needs to be studied further. Also, instead of fixed thresholds, the performance of the protocol with variable threshold values that could be set adaptively according to network conditions, needs to be evaluated.

\section{REFERENCES}

[1] I. Chlamtac, M. Conti, J. Jennifer, N. Liu, 2003 'Mobile ad hoc networking: imperatives and challenges' Ad Hoc Networks, Vol. 1, pp: 13-64.

[2] T. Clausen, P. Jacquet, A. Laouiti, P. Minet, P. Muhlethaler, A. Qayyum, and L. Viennot, 2003, "Optimized link state routing protocol", Internet Draft: http://www.ietf.org/rfc/rfc3626.txt, October 2003.

[3] C. E. Perkins and P. Bhagwat, 1994, Highly Dynamic Destination Sequenced Distance Vector Routing (DSDV) for Mobile Computers, In Proc. of ACM SIGCOMM'94, pp. 234-244, Sept. 1994, London, UK.

[4] J. J. Garcia-Luna-Aceves and M. Spohn, 1999, SourceTree Routing in Wireless Networks, In Proc. of IEEE ICNP'99, Nov. 1999

[5] C. E. Perkins and E. M Royer, 1999, Ad hoc On-Demand Distance Vector Routing, In Proc. of $2^{\text {nd }}$ IEEE workshop on Mobile Computing Systems and Applications (WMCSA ‘99), pp. 90-100, Feb 1999, New Orleans, LA.

[6] D. B. Johnson and D. A. Maltz, 1996, "Dynamic Source Routing in Ad hoc Wireless Networks", Mobile Computing, Vol. 5, pp. 153-181, 1996.

[7] V. D. Park and M. S. Corson, 1997, A Highly Adaptive Distributed Routing Algorithm for Mobile Wireless Networks, In Proc. of IEEE Conference on Computer Communications INFOCOM '97, pp. 1405-1413, Kobe, Japan, Apr. 1997.

[8] S. Mueller, R. P. Tsang, D. Ghosal, 2004, "Multipath Routing in Mobile Ad Hoc Networks: Issues and Challenges", invited paper in Lecture Notes in Computer
Science, Edited by Maria Carla Calzarossa and Erol Gelenbe, 2004.

[9] R. Kumar, A. K Sarje and M. Misra, 2010, “An AODV based QoS Routing Protocol for Delay Sensitive Applications in Mobile Ad Hoc Networks", Journal of Digital Information Management, Vol 8 No. 5, October 2010.

[10]J. Y. Choi and Y. B. Ko, 2010, Multi-path Routing with Load-aware Metric for Tactical Ad Hoc Networks, International Conference on Information and Communication Technology Convergence (ICTC), Jeju, 17-19, Nov, 2010.

[11] O. Souihli, M. Frikha and M. B Hamouda, 2009, "Loadbalancing in MANET shortest-path routing protocols", in proc. of Journal on Ad Hoc Networks, vol. 7, no. 2, pp: 431- 442, March 2009.

[12] S. P. Terdal, V. D. Mytri, A. Damodaram, B. Salimath, 2011, "Multipath Load Balancing : An Entropy based Clustering Solution for Mobile Ad hoc Networks", International Journal on Recent Trends in Engineering \& Technology, Vol. 05, No. 01, Mar 2011.

[13] L. R. Reddy and S.V. Raghavan, 2007, "SMORT: Scalable multipath on-demand routing for mobile ad hoc networks", in proc. of Journal on Ad Hoc Networks, vol. 5, no. 2, pp: 162- 188, March 2007.

[14] S. Kumar, V. S. Raghavan, J. Deng, 2006, "Medium Access Control protocols for ad hoc wireless networks: A survey", Ad hoc networks 4 (3) (2006), pp: 326-358.

[15] P. Mohapatra, J. Li, C. Gui, 2003, "QoS in Mobile Ad hoc Networks", IEEE Wireless Communications, June 2003, pp: 45-52.

[16] G. I. Ivascu, S. Pierre, A. Quintero, 2009, "QoS routing with traffic distribution in mobile ad hoc networks", in proc. of Journal on Computer Communications, vol. 32, no.2, pp: 305 - 316, February 2009.

[17] Q. Xue, A. Ganz, 2003, “Ad hoc QoS on-demand routing (AQOR) in mobile ad hoc networks", Journal of Parallel Distributed Computing Vol. 63 (2003), pp. 154-165.

[18] C. R. Lin, J. S. Liu, 1999, "QoS Routing in Ad Hoc Wireless Networks", IEEE Journal on Selected Areas in Communications, Vol. 17, No. 8, August 1999, pp. 14261438.

[19] C. Mbarushimana, A. Shahrabi, 2008, TSLA: A QoSAware On-Demand Routing Protocol for Mobile Ad Hoc Networks, In Proc. of $7^{\text {th }}$ International Conference AD HOC NOW, September 2008, pp. 265-278.

[20] M. K Marina, S. R Das, 2001, On Demand Multipath Distance Vector Routing in Ad hoc Networks, in Proc. of the Ninth International Conference on Network Protocols, pp: 14-23, 2001.

[21] W. H. Liao, Y. C. Tseng, S. L. Wang and J. P. Sheu, A Multipath QoS Routing Protocol in a Wireless Mobile Ad Hoc Network, IEEE International Conference on Networking (ICN), 2001.

[22] V. Rishiwal, S. Verma and S. K. Bajpai, 2009, "A QoS Based Power Aware Routing in MANETs" International 
Journal of Computer Theory and Engineering, Vol. 1, No. 1, pp: 47-54, April 2009.

[23] F. Qin and Y. Liu, 2009, Multipath Routing for Mobile Ad Hoc Network, Proc. of the 2009 International Symposium on Information Processing (ISIP'09) Huangshan, P. R. China, pp. 237-240, August 21-23, 2009.

[24] S. T Sheu, J. Chen, 2001, A Novel Delay Oriented Shortest Path Routing Protocol for Mobile Ad Hoc Networks, ICC 2001, The IEEE International Conference on Communications, Vol 9, pp 1930-34, June 2001.

[25] X. Gao, X. Zhang, D. Shi, F. Zou and W. Zhu, 2007, Contention and Queue-aware Routing Protocol for Mobile Ad hoc Networks, International Conference on Wireless Communications, Networking and Mobile Computing (WiCom 2007), Shanghai, 21-25 Sept. 2007.

[26] Network Simulator, http://www.isi.edu/nsnam/ns, Accessed, $31^{\text {st }}$ June 2013 